\title{
Previous chronic symptomatic and asymptomatic cerebral hemorrhage in patients with acute ischemic stroke
}

\author{
Ge-Fei Li ${ }^{1,2} \cdot$ Yi-Lan Wu ${ }^{1,2} \cdot$ Shuo Wang ${ }^{1} \cdot$ Yan-Hui Shi ${ }^{1,2} \cdot$ Rong Zhao ${ }^{1,2} \cdot$ Feng-Di Liu ${ }^{1,2} \cdot$ Yi-Sheng Liu ${ }^{1,2}$. \\ Mei-Ting Zhuang ${ }^{1,2} \cdot$ Ying Zhao $^{1,2} \cdot$ Qi Sun ${ }^{3}$. Guo-Hong Cui ${ }^{1}$ • Jian-Ren Liu ${ }^{1} \mathbb{D}$
}

Received: 29 September 2018 / Accepted: 19 November 2018 / Published online: 28 November 2018

(C) The Author(s) 2018

\begin{abstract}
Purpose Identifying previous chronic cerebral hemorrhage ( $\mathrm{PCH})$, especially asymptomatic cases in patients with ischemic stroke, is essential for proper antithrombotic management. The study aimed to further clarify the prevalence of $\mathrm{PCH}$ and the associated factors in patients with acute ischemic stroke using multi-modal neuroimaging including susceptibility-weighted MR imaging (SWI).

Methods This was a retrospective cross-sectional study of 382 patients with acute ischemic stroke. All patients underwent 3.0-T MRI for cranial SWI, 1.5-T or 3.0-T conventional cranial MRI, and cranial CT. Patients found with PCH were matched 1:4 with patients without $\mathrm{PCH}$. Clinical manifestation, computed tomography, conventional cranial MRI, and cranial SWI were used to determine $\mathrm{PCH}$. Clinical and neuroimaging findings between the patients with symptomatic vs. asymptomatic PCH were compared.

Results Thirty-six patients $(36 / 382,9.4 \%)$ were determined to have had a PCH. Of these 36 patients, 17 (17/36, 47.2\%, or $17 /$ $382,4.5 \%)$ had asymptomatic PCH. Multivariable analysis showed that serum total cholesterol $(\mathrm{OR}=0.510,95 \% \mathrm{CI} 0.312$ $0.832, P=0.007)$, cerebral microbleeds $(\mathrm{OR}=6.251,95 \% \mathrm{CI} 2.220-17.601, P=0.001)$, and antithrombotic drugs history $(\mathrm{OR}=$ $3.213,95 \%$ CI 1.018-10.145, $P=0.047$ ) were independently associated with PCH. Asymptomatic PCH had similar clinical and neuroimaging characteristics with symptomatic $\mathrm{PCH}$.

Conclusion PCH is not uncommon in acute ischemic stroke patients. Total serum cholesterol, cerebral microbleeds on SWI, and history of antithrombotic drugs were independently associated with $\mathrm{PCH}$ in patients with acute ischemic stroke. Asymptomatic $\mathrm{PCH}$, which is easier to be missed and has similar characteristics with symptomatic $\mathrm{PCH}$, should draw much attention.
\end{abstract}

Keywords Ischemic stroke · Susceptibility-weighted MR imaging $\cdot$ Previous chronic cerebral hemorrhage

Electronic supplementary material The online version of this article (https://doi.org/10.1007/s00234-018-2141-y) contains supplementary material, which is available to authorized users.

Jian-Ren Liu

liujr021@sjtu.edu.cn

1 Department of Neurology, Comprehensive Stroke Center, Shanghai Ninth People's Hospital, Shanghai Jiao Tong University School of Medicine, 639 Zhizaoju Road, Huangpu District, Shanghai 200011, China

2 Clinical Research Center, Shanghai Ninth People's Hospital, Shanghai Jiao Tong University School of Medicine, Shanghai 200011, China

3 Department of Radiology, Shanghai Ninth People's Hospital, Shanghai Jiao Tong University School of Medicine, Shanghai 200011, China

\section{Introduction}

Patients with cerebral hemorrhage frequently have indications for antithrombotic therapy. This represents a therapeutic dilemma as cerebral hemorrhage is considered a contraindication to antithrombotic medication. There were no long-term randomized studies addressing this issue. Some acute ischemic stroke patients may have had a previous chronic cerebral hemorrhage ( $\mathrm{PCH})$ that could recur with the use of antithrombotic therapy for secondary prevention, leading to poorer outcomes [1-7].

Susceptibility-weighted MR imaging (SWI) is more sensitive to fresh or chronic cerebral hemorrhagic lesions than conventional MRI and allows the detection of $\mathrm{PCH}$, especially for asymptomatic one that was easily to be misdiagnosed [8]. Identifying PCH in patients with acute ischemic stroke could 
be essential for prudent initiation of antithrombotic therapy, esp., intensive antithrombotic therapy. However, the prevalence of $\mathrm{PCH}$ and proper method to detect asymptomatic or symptomatic $\mathrm{PCH}$ needs to be further clarified for acute ischemic stroke patients. Therefore, this study used SWI examination, combined with multi-modal neuroimaging examinations and detailed clinical data, to observe the prevalence of PCH (symptomatic or not) and analyze the associated factors of $\mathrm{PCH}$ in patients admitted for acute ischemic stroke in a comprehensive stroke center.

\section{Methods}

\section{Patients}

This retrospective cross-sectional study analyzed patients who were diagnosed with acute ischemic stroke and admitted at the stroke center of our hospital, one of national comprehensive stroke centers between 12/12/2011 and 3/6/2013. This study was approved by the ethic committee of our hospital. Informed consent was waived by the committee because of the retrospective nature of the study. The patient inclusion criteria are presented in the Supplemental Material.

\section{Neuroimaging}

All patients underwent $\mathrm{CT}$ in the emergency room to exclude acute cerebral hemorrhage. Conventional MRI and diffusionweighted imaging (DWI) were completed within 5 days of admission. SWI was performed within 10 days of admission. Examinations were blindly reviewed by two experienced neurologists. A third senior physician was invited for consensus in case of controversy between the first two physicians. Details about the MRI examinations are provided in the Supplemental Material.

Cranial SWI were used to observe and analyze cerebral microbleeds (CMBs) $[8,9]$. According to the Microbleed Anatomical Rating Scale (MARS) [10], CMB was defined as circular or oval low signal lesions with clear boundary on SWI sequence, with a diameter of 2-10 mm, while excluding lesions with similar imaging manifestations (including calcification, ferruginous sediment, cavernous hemangioma.) by combining with phase diagrams, mIP, other MRI sequences $[11,12]$, and cranial CT. The amount of CMBs was recorded according to the MARS for quantitative evaluation [10].

The presence of $\mathrm{PCH}$ was determined based on nonenhanced cranial CT, conventional cranial MRI scanning (routine scanning sequence), cranial SWI examination, and disease history. PCH referred to hypointensity on SWI magnitude images, with a diameter $>1 \mathrm{~cm}$. They appeared as softened hypodense lesions on cranial CT, hypointensity on T1weighted MRI, and hyperintensity on T2-weighted images, and on MRI (T1 - or T2-weighted MRI, or FLAIR MRI) there would be a hypointense rim around the lesion, indicating iron deposition after absorption of the hematoma. In addition, cerebral hemorrhage could be differentiated from calcification using SWI filtered phase image; in filtered phase images for a left-handed system, calcification has low signal intensity, while cerebral hemorrhage has high signal intensity [11, 12]. Previous neuroimaging studies were checked as possible for patients with a medical history of cerebral hemorrhage. Besides cranial CT scan, all MRI sequences (DWI, T1weighted MRI, T2-weighted MRI, FLAIR, and SWI) were carefully reviewed to further exclude acute intracerebral hemorrhages, and those suspected transformation hemorrhage related to the acute ischemic stroke on cranial MRI would not be defined as $\mathrm{PCH}$.

\section{Classification of acute stroke}

Stroke was classified according to the TOAST (Trial of org 10172 in Acute Stroke Treatment) classification [13] and combined with disease history and imaging characteristics of the patients, and acute ischemic stroke was classified into five types: large-artery atherosclerosis (LAA), small-artery occlusion (SAA), cardiogenic embolism (CE), stroke of other demonstrated etiology (SOE), and stroke of other undemonstrated etiology (SUE). Hemorrhagic transformation during acute stroke was defined as the development of a hemorrhage after an acute ischemic stroke.

\section{Statistical analysis}

Continuous data was expressed as mean \pm standard deviation or median (range), and analyzed with the Student $t$ test or the Wilcoxon rank sum test, as appropriate. Categorical data was expressed as frequencies and percentages and analyzed with the Pearson chi-square test or Fisher exact test, as appropriate. Subsequently, each variable was introduced into a logistic regression model. Factors with $P$ values $<0.05$ in univariable analyses were entered into the multivariable logistic regression model, screened using the backward method, where the inclusion criterion was $P<0.05$. Statistical analyses were performed using SPSS 19.0 (IBM, Armonk, NY, USA). Twosided $P$ values $<0.05$ were considered statistically significant.

\section{Results}

Thirty-six patients $(36 / 382,9.4 \%)$ had PCH (Supplementary Table S1). Of these, $17(17 / 36,47.2 \%$, or $17 / 382,4.5 \%)$ had asymptomatic PCH. Multivariable analysis showed that serum total cholesterol $(\mathrm{OR}=0.510,95 \% \mathrm{CI} 0.312-0.832, P=$ $0.007)$, cerebral microbleeds $(\mathrm{OR}=6.251,95 \%$ CI 2.220 $17.601, P=0.001)$, and antithrombotic drugs history $(\mathrm{OR}=$ 
Table 1 Univariable logistic regression of factors associated with cerebral hemorrhage

\begin{tabular}{|c|c|c|c|}
\hline & Odds ratio & $95 \%$ confidence interval & $P$ \\
\hline \multicolumn{4}{|l|}{ TOAST classification } \\
\hline LAA vs. SUE & 1.011 & $0.248-4.117$ & 0.988 \\
\hline SAA vs. SUE & 0.383 & $0.121-1.214$ & 0.103 \\
\hline CE vs. SUE & - & - & - \\
\hline SOE vs. SUE & 1.386 & $0.470-3.517$ & 0.625 \\
\hline Hypertension history & 1.843 & $0.664-5.119$ & 0.241 \\
\hline Hypertension classification & 1.233 & $0.887-1.713$ & 0.212 \\
\hline SBP & 1.000 & $0.983-1.018$ & 0.969 \\
\hline DBP & 0.998 & $0.966-1.032$ & 0.927 \\
\hline Diabetes & 1.415 & $0.675-2.967$ & 0.358 \\
\hline Fasting blood glucose & 1.039 & $0.901-1.196$ & 0.600 \\
\hline $\mathrm{HbAlc}$ & 1.164 & $0.969-1.400$ & 0.105 \\
\hline LDL & 0.511 & $0.313-0.836$ & 0.008 \\
\hline HDL & 0.623 & $0.155-2.507$ & 0.505 \\
\hline $\mathrm{TC}$ & 0.498 & $0.318-0.778$ & 0.002 \\
\hline TG & 1.006 & $0.616-1.642$ & 0.980 \\
\hline Uric acid & 1.003 & $0.999-1.007$ & 0.100 \\
\hline Creatinine & 1.011 & $0.997-1.025$ & 0.133 \\
\hline Microbleeds & 7.000 & $2.742-17.867$ & $<0.001$ \\
\hline Microbleeds score & 1.062 & $1.006-1.121$ & 0.030 \\
\hline Hemorrhagic transformation & 0.265 & $0.034-2.078$ & 0.206 \\
\hline Prominent vessel sign on SWI & 3.086 & $0.841-11.328$ & 0.089 \\
\hline History of antithrombotic drugs & 3.143 & $1.176-8.400$ & 0.022 \\
\hline Brain white matter degeneration score & 1.357 & $1.090-1.690$ & 0.006 \\
\hline
\end{tabular}

TOAST Trial of Org10172 in Acute Stroke Treatment, LAA large-artery atherosclerosis, SAA small-artery occlusion, $C E$ cardio-embolism, $S O E$ stroke of other demonstrated etiology, $S U E$ stroke of other undemonstrated etiology, $S B P$ systolic blood pressure, $D B P$ diastolic blood pressure, $L D L$ low-density lipoprotein, $H D L$ highdensity lipoprotein, $T C$ total cholesterol, $T G$ triglycerides
$3.213,95 \%$ CI $1.018-10.145, P=0.047)$ were independently associated with PCH (Tables 1 and 2). Asymptomatic PCH had similar demographic, neuroimaging, and biochemical characteristics with symptomatic PCH (Supplementary Table S2).

\section{Discussion}

The study aimed to analyze the prevalence of $\mathrm{PCH}$ in patients with acute ischemic stroke using SWI and to analyze the factors associated with $\mathrm{PCH}$ in those patients. Thirty-six (9.4\%) patients were found to have $\mathrm{PCH}$. Multivariable analysis results showed that TC, existence of CMBs on SWI, and medication history of antithrombotic drugs were independently associated with the presence of a $\mathrm{PCH}$ in patients with ischemic stroke. There were no differences in clinical characteristics between previous symptomatic and asymptomatic $\mathrm{PCH}$.

In the present study, the frequency of $\mathrm{PCH}$ was $9.4 \%$, or $4.5 \%$ for asymptomatic $\mathrm{PCH}$, which was slightly lower than in previous studies $[14,15]$. The discrepancies could be due to the selection of the subjects and geographical regions, as well as to different imaging protocols.

Patients with ischemic stroke should promptly receive antithrombotic therapy for secondary prevention if they do not have any significant contraindication, but for those with both
Table 2 Multivariable regression analysis of factors associated with cerebral hemorrhage

\begin{tabular}{llll}
\hline & Odds ratio & $95 \%$ confidence interval & $P$ \\
\hline Total cholesterol & 0.510 & $0.312-0.832$ & 0.007 \\
Cerebral microbleeds & 6.251 & $2.220-17.601$ & 0.001 \\
Use history of antithrombotic drugs & 3.213 & $1.018-10.145$ & 0.047 \\
\hline
\end{tabular}

Factors with $P$ values $<0.05$ in univariable analyses were tested using a backward method 
acute ischemic stroke and $\mathrm{PCH}$, symptomatic or not, longterm aggressive antithrombotic therapy should be used with caution after considering the risks and benefits to avoid rebleeding [3-7]. There were no long-term randomized studies addressing this issue. There is a marked paucity of evidence to guide clinicians when planning the long-term management of patients with cerebral hemorrhage and cogent indications for antithrombotic therapy. Recently, a randomized controlled trial, "The REstart or STop Antithrombotics Randomised Trial (RESTART) after stroke due to intracerebral haemorrhage," has been registered to compare starting versus avoiding antiplatelet drugs for adults surviving antithrombotic-associated ICH at 122 hospital sites in the UK [16].

$\mathrm{CMB}$ can be associated with poor prognosis of stroke [17, 18]. A recent study reported in White and Chinese patients with $\geq 5$ CMBs withholding antiplatelet drugs during the first year after transient ischemic attack/ischemic stroke may be inappropriate; however, the risk of ICH may outweigh any benefit thereafter [19].

The present study found that there were more patients suffering from CMBs in patients with $\mathrm{PCH}$ compared with patients without $\mathrm{PCH}$, and $\mathrm{CMBs}$ independently associated with existence of $\mathrm{PCH}$. Thus, for patients with ischemic stroke, it could be necessary to perform SWI examination to promptly discover CMBs, as well as $\mathrm{PCH}$, in order to guide antithrombotic medication.

The proper management of patients with acute ischemic stroke and PCH remains controversial [3-7, 20, 21]. Our study found the $\mathrm{PCH}$ was not uncommon in acute ischemic stroke patients and antithrombotic medication should be used with more caution after considering risk and benefit. The present study also suggests that there were no differences in clinical characteristics between symptomatic and asymptomatic $\mathrm{PCH}$, making the latter easy to be missed, which could influence the outcomes of antithrombotic therapy and increase the risk of re-bleeding. The present study suggests that SWI could be a proper imaging modality for identifying patients with asymptomatic $\mathrm{PCH}$, hence guiding the therapeutic strategy and patient management.

The limitations are presented as Supplemental Material.

\section{Conclusion}

In this study of patients admitted in national stroke centers for acute ischemic stroke and who underwent conventional MRI and SWI and cranial CT, 9.4\% were found to have a PCH. Total cholesterol, the presence of CMBs on SWI, and history of antithrombotic drugs were independently associated with the presence of $\mathrm{PCH}$ in patients with acute ischemic stroke. Asymptomatic $\mathrm{PCH}$, which is easier to be missed but has similar characteristics of demographic, neuroimaging, and serum biochemical testing with symptomatic $\mathrm{PCH}$, should draw much attention. Hence, implementing routine cranial SWI examination could show PCH more sensitively, allowing the optimal management of these patents in order to prevent rebleeding and worsening of the clinical outcomes.

\section{Compliance with ethical standards}

Funding This study was funded by the "Prevention and Control of Chronic Diseases Project" of Shanghai Hospital Development Center [No. SHDC12015310 to JRL], the SHSMU-ION Research Center for Brain Disorders [No. 2015NKX006 to JRL], the Shanghai Municipal Education Commission-Gaofeng Clinical Medicine Grant Support [No. 20161422 to JRL], the Clinical Research Project from Shanghai Jiao Tong University School of Medicine [No. DLY201614 to JRL] and the Biomedicine Key program from Shanghai Municipal Science and Technology Commission [No. 16411953100 JRL].

Conflict of interest The authors declare that they have no conflict of interest.

Ethical approval All procedures performed in the studies involving human participants were in accordance with the ethical standards of the institutional and/or national research committee and with the 1964 Helsinki Declaration and its later amendments or comparable ethical standards. For this type of study formal consent is not required.

Informed consent For this type of retrospective study formal consent is not required.

Open Access This article is distributed under the terms of the Creative Commons Attribution 4.0 International License (http:// creativecommons.org/licenses/by/4.0/), which permits unrestricted use, distribution, and reproduction in any medium, provided you give appropriate credit to the original author(s) and the source, provide a link to the Creative Commons license, and indicate if changes were made.

\section{References}

1. Inohara T, Xian Y, Liang L, Matsouaka RA, Saver JL, Smith EE, Schwamm LH, Reeves MJ, Hernandez AF, Bhatt DL, Peterson ED, Fonarow GC (2018) Association of intracerebral hemorrhage among patients taking non-vitamin $\mathrm{K}$ antagonist vs vitamin $\mathrm{K}$ antagonist oral anticoagulants with in-hospital mortality. JAMA 319(5):463-473. https://doi.org/10.1001/jama.2017.21917

2. Tsivgoulis G, Lioutas VA, Varelas P, Katsanos AH, Goyal N, Mikulik R, Barlinn K, Krogias C, Sharma VK, Vadikolias K, Dardiotis E, Karapanayiotides T, Pappa A, Zompola C, Triantafyllou S, Kargiotis O, Ioakeimidis M, Giannopoulos S, Kerro A, Tsantes A, Mehta C, Jones M, Schroeder C, Norton C, Bonakis A, Chang J, Alexandrov AW, Mitsias P, Alexandrov AV (2017) Direct oral anticoagulant- vs vitamin K antagonist-related nontraumatic intracerebral hemorrhage. Neurology 89(11):11421151. https://doi.org/10.1212/WNL.0000000000004362

3. Gulati S, Solheim O, Carlsen SM, Øie LR, Jensberg H, Gulati AM, Madsbu MA, Giannadakis C, Jakola AS, Salvesen Ø (2018) Risk of intracranial hemorrhage (RICH) in users of oral antithrombotic drugs: Nationwide pharmacoepidemiological study. PLoS One 13(8):e0202575. https://doi.org/10.1371/journal.pone.0202575 
4. Perry LA, Berge E, Bowditch J, Forfang E, Rønning OM, Hankey GJ, Villanueva E, Al-Shahi Salman R (2017) Antithrombotic treatment after stroke due to intracerebral haemorrhage. Cochrane Database Syst Rev 5:CD012144. https://doi.org/10.1002/ 14651858.CD012144.pub2

5. Roquer J, Vivanco Hidalgo RM, Ois A, Rodríguez Campello A, Cuadrado Godia E, Giralt Steinhauer E, Gómez González A, Soriano-Tarraga C, Jiménez Conde J (2017) Antithrombotic pretreatment increases very-early mortality in primary intracerebral hemorrhage. Neurology 88(9):885-891. https://doi.org/10.1212/ WNL.0000000000003659

6. Pasquini M, Charidimou A, van Asch CJ, Baharoglu MI, Samarasekera N, Werring DJ, Klijn CJ, Roos YB, Al-Shahi Salman R, Cordonnier C (2014) Variation in restarting antithrombotic drugs at hospital discharge after intracerebral hemorrhage. Stroke 45(9):2643-2648. https://doi.org/10.1161/STROKEAHA. 114.006202

7. Giakoumettis D, Alexiou GA, Vrachatis DA, Themistoklis K, Stathis P, Vavuranakis M, Themistocleous MS (2017) Antithrombotic treatment management in patients with intracerebral hemorrhage: reversal and restart. Curr Pharm Des 23(9):13921405. https://doi.org/10.2174/1381612822666161205111459

8. Thomas B, Somasundaram S, Thamburaj K, Kesavadas C, Gupta AK, Bodhey NK, Kapilamoorthy TR (2008) Clinical applications of susceptibility weighted MR imaging of the brain - a pictorial review. Neuroradiology 50(2):105-116

9. Tao XX, Li GF, Wu YL, Liu YS, Zhao Y, Shi YH, Zhuang MT, Hou TY, Zhao R, Liu FD, Wang XM, Shen Y, Cui GH, Su JJ, Chen W, Tang XM, Sun J, Liu JR (2017) Relationship between intracranial internal carotid artery calcification and enlarged cerebral perivascular space. Neuroradiology 59(6):577-586. https://doi. org/10.1007/s00234-017-1838-7

10. Gregoire SM, Chaudhary UJ, Brown MM, Yousry TA, Kallis C, Jager HR, Werring DJ (2009) The Microbleed Anatomical Rating Scale (MARS): reliability of a tool to map brain microbleeds. Neurology 73(21):1759-1766. https://doi.org/10.1212/WNL. 0b013e3181c34a7d

11. Ayaz M, Boikov AS, Haacke EM, Kido DK, Kirsch WM (2010) Imaging cerebral microbleeds using susceptibility weighted imaging: one step toward detecting vascular dementia. J Magn Reson Imaging 31(1):142-148. https://doi.org/10.1002/jmri.22001

12. Haacke EM, Mittal S, Wu Z, Neelavalli J, Cheng YC (2009) Susceptibility-weighted imaging: technical aspects and clinical applications, part 1. AJNR Am J Neuroradiol 30(1):19-30. https://doi. org/10.3174/ajnr.A1400

13. Adams HP Jr, Bendixen BH, Kappelle LJ, Biller J, Love BB, Gordon DL, Marsh EE 3rd (1993) Classification of subtype of acute ischemic stroke. Definitions for use in a multicenter clinical trial. TOAST. Trial of Org 10172 in Acute Stroke Treatment. Stroke 24(1):35-41

14. Aoki J, Shibazaki K, Saji N, Uemura J, Sakamoto Y, Kimura K (2014) Risk of intracerebral hemorrhage after thrombolysis in patients with asymptomatic hemorrhage on T2*. Cerebrovasc Dis 38(2):107-116. https://doi.org/10.1159/000365207

15. Lee SH, Kim BJ, Han MK, Park TH, Lee KB, Lee BC, Yu KH, Oh MS, Cha JK, Kim DH, Nah HW, Lee J, Lee SJ, Ko Y, Kim JG, Park JM, Kang K, Cho YJ, Hong KS, Choi JC, Kim JT, Choi K, Kim DE, Ryu WS, Kim WJ, Shin DI, Yeo M, Lee J, Lee JS, Gorelick PB, Bae HJ (2016) Should we exclude acute stroke patients with previous intracerebral hemorrhage from receiving intravenous thrombolysis? Int J Stroke 11(7):783-790. https://doi.org/10.1177/ 1747493016654289

16. Al-Shahi Salman R, Dennis MS, Murray GD, Innes K, Drever J, Dinsmore L, Williams C, White PM, Whiteley WN, Sandercock PAG, Sudlow CLM, Newby DE, Sprigg N, Werring DJ, RESTART collaborators (2018) The REstart or STop Antithrombotics Randomised Trial (RESTART) after stroke due to intracerebral haemorrhage: study protocol for a randomised controlled trial. Trials 19(1):162. https://doi.org/10.1186/s13063-018-2542-6

17. Wang DN, Hou XW, Yang BW, Lin Y, Shi JP, Wang N (2015) Quantity of cerebral microbleeds, antiplatelet therapy, and intracerebral hemorrhage outcomes: a systematic review and meta-analysis. J Stroke Cerebrovasc Dis 24(12):2728-2737. https://doi.org/10. 1016/j.jstrokecerebrovasdis.2015.08.003

18. Chacon-Portillo MA, Llinas RH, Marsh EB (2018) Cerebral microbleeds shouldn't dictate treatment of acute stroke: a retrospective cohort study evaluating risk of intracerebral hemorrhage. BMC Neurol 18(1):33. https://doi.org/10.1186/s12883-018-1029-0

19. Lau KK, Lovelock CE, Li L, Simoni M, Gutnikov S, Küker W, Mak HKF, Rothwell PM (2018) Antiplatelet treatment after transient ischemic attack and ischemic stroke in patients with cerebral microbleeds in 2 large cohorts and an updated systematic review. Stroke 49(6):1434-1442. https://doi.org/10.1161/STROKEAHA. 117.020104

20. Pennlert J, Asplund K, Carlberg B, Wiklund PG, Wisten A, Asberg S, Eriksson M (2015) Antithrombotic treatment following intracerebral hemorrhage in patients with and without atrial fibrillation. Stroke 46(8):2094-2099. https://doi.org/10.1161/STROKEAHA. 115.009087

21. Use of Ottosen TP, Grijota M, Hansen ML, Brandes A, Damgaard D, Husted SE, Johnsen SP (2016) Antithrombotic therapy and longterm clinical outcome among patients surviving intracerebral hemorrhage. Stroke 47(7):1837-1843. https://doi.org/10.1161/ STROKEAHA.116.012945 